\title{
A Novel Fiber-Laser-Based Sensor Network With Self-Healing Function
}

\author{
Peng-Chun Peng, Hong-Yih Tseng, and Sien Chi
}

\begin{abstract}
In this letter, we present a novel fiber-laser-based sensor network with self-healing function and demonstrate its effectiveness. The network survivability and capacity for a multipoint sensor system are enhanced by adding switches in a self-healing ring architecture. The fiber laser adopted in this system is a linear-cavity fiber Bragg grating (FBG) laser leading to the signal-to-noise ratio over $52 \mathrm{~dB}$ for the sensor network. The network survivability of a ten-point FBG sensor is experimentally examined. The experimental results show that the proposed system can facilitate reliable sensing network for a large-scale and multipoint smart structure.
\end{abstract}

Index Terms-Fiber Bragg grating (FBG), fiber laser, fiber sensor, self-healing function, sensor network.

\section{INTRODUCTION}

$\mathbf{F}$ IBER BRAGG grating (FBG) sensors have been identified as very important sensing elements, especially for the strain measurement in smart structures [1]-[4]. In many applications, the arrays of FBG sensors are required for multipoint or distributed measurement. The multiplexing of FBG sensors is therefore essential to reduce the cost per sensing point and to increase the competitiveness of FBG sensors against the conventional electrical sensors. The most popular technique for multiplexing FBG sensors is the wavelength-division-multiplexing (WDM) technique. A large-scale FBG sensor system with 60 sensors has been experimentally demonstrated by using a combination of WDM and time-division-multiplexing (TDM) techniques [1]. Such research associated with sensing network survivability would be an ongoing challenge for the practical applications of the multipoint FBG sensor systems. Therefore, enhancing the survivability of an FBG sensor network is very important. However, the in-line topology, star topology, and tree topology generally adopted in an FBG sensor network cannot protect the sensing network [5]. Moreover, a broad-band light source is usually used in an FBG sensor system. Because the optical power reflected from an FBG is weak, the sensing resolution and the capacity of a fiber sensor system would be limited by the broad-band light source.

In recent years, the ring topology based on electrical add-drop multiplexers (ADMs) for the SDH/SONET has been widely applied in a fiber network where high reliability and low cost are required [6]. In this letter, we present a novel FBG

Manuscript received August 13, 2002; revised September 27, 2002. This work was supported by the Academic Excellence Program of R.O.C. Ministry of Education under Contract 90-E-FA06-1-4-90X023.

The authors are with the Institute of Electro-Optical Engineering, National Chiao-Tung University, Hsinchu 300, Taiwan, R.O.C. (e-mail: pcpeng.eo90g@nctu.edu.tw).

Digital Object Identifier 10.1109/LPT.2002.806088

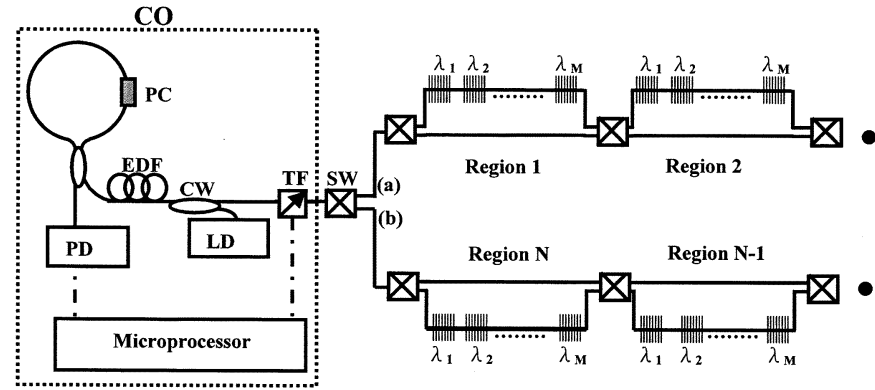

Fig. 1. Schematic diagram of the proposed self-healing sensor network based on a linear-cavity fiber laser scheme LD: $980 \mathrm{~nm} . \lambda_{i}$ : FBG. C1: $2 \times 2$ coupler. SW: Switch. CW: WDM coupler. CO: Center office.

sensor network with a self-healing function to increase the reliability of the sensing network. The self-healing function is constructed by using the ring topology. A $1 \times 2$ switch in the ring topology is used to check the breakpoint and $2 \times 2$ switches are used to enhance the sensing capacity in the sensor network. Such a simple self-healing function for the ring topology can support real-time monitoring and reveal the sudden breakpoint of the fiber link. Furthermore, we use a linear-cavity fiber laser scheme for our proposed sensor system. This fiber-laser-based sensing network can avoid the reduction of the signal-to-noise ratio (SNR) because of the low-power broad-band source together with its amplified spontaneous emission (ASE) noise. The benefits of our proposed fiber-laser-based sensor system can facilitate reliable sensing network for a large-scale and multipoint smart structure.

\section{PRINCIPLE}

Fig. 1 shows the proposed configuration of the self-healing FBG sensor network. The FBG sensor system consists of the sensing FBG network and a central office providing the light source and discriminating the signals from the sensing network. The light source in this system is a linear-cavity fiber laser that comprises a fiber loop mirror, the sensing FBG $\left(\lambda_{1}, \lambda_{2}, \ldots\right.$, or $\left.\lambda_{M}\right)$ simultaneously acting as another cavity mirror, a polarization controller (PC), a tunable bandpass filter (TF), and a section of erbium-doped fiber (EDF) pumped by a 980-nm laser diode (LD). The ring architecture for this sensing network is composed of the sensing FBGs $\left(\lambda_{1} \sim \lambda_{M}\right)$, a piece of single-mode fiber (SMF), and a $2 \times 2$ switch. $N$ groups of such a ring subnetwork (Sensing Region $1 \sim$ Sensing Region N) can be cascaded one by one for constructing a large-scale ring architecture. The $2 \times 2$ switch in each subnetwork is controlled by a TDM signal to enhance the capacity for multipoint sensing. For example, the dashed line in Fig. 2 schematically indicates 


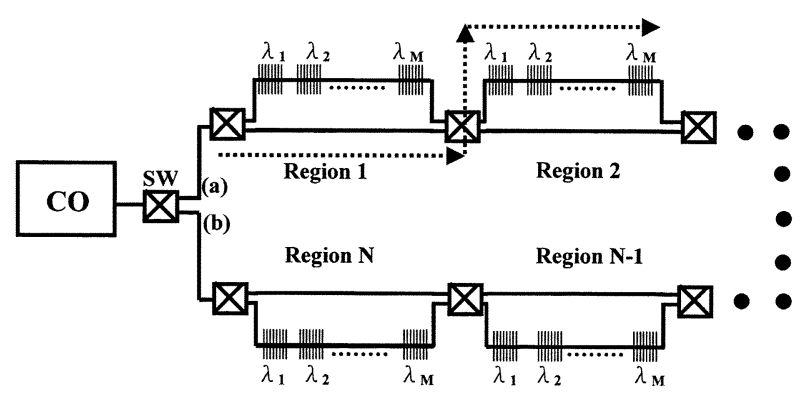

Fig. 2. Schematic situation (indicated by the dashed line) when Sensing Region 2 is selected by using a TDM signal.

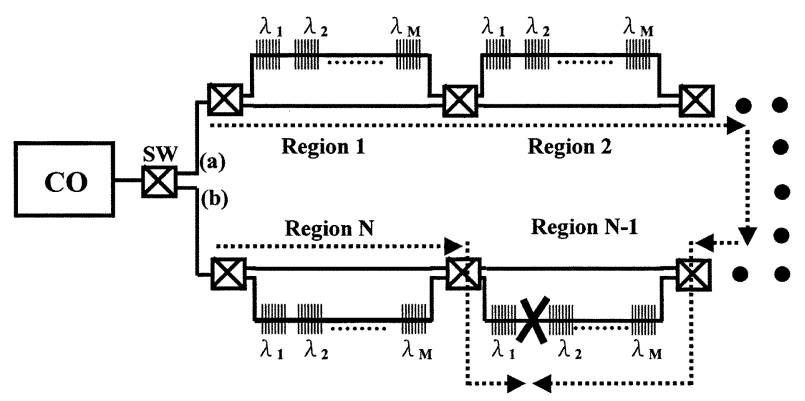

Fig. 3. Schematic situation when a breakpoint occurs and the fiber link fails in Sensing Region N-1.

the situation when Sensing Region 2 is selected by using the TDM signal. Because of this ring topology incorporated with the TDM technology, the proposed system can support a large number of sensor elements. Furthermore, for such a large-scale sensing network, we consider the self-healing function for the ring architecture by controlling the $1 \times 2$ switch. Such a self-healing architecture can offer survival function under link failure by reconfiguring the fiber network. Fig. 3 schematically shows a situation when the fiber link fails in Sensing Region $\mathrm{N}-1$. When the $1 \times 2$ switch is in state (a), we lose the sensing signal from $\operatorname{FBG}\left(\lambda_{1}\right)$ in Region $\mathrm{N}-1$. Nevertheless, the $1 \times 2$ switch can be modified to state (b) to reconfigure the sensing signal from $\operatorname{FBG}\left(\lambda_{1}\right)$ in Region $\mathrm{N}-1$. The drawback of this self-healing function for the sensing network protection is that all the switches in the network induce extra loss and further reduce the SNR in the system. In order to enhance the SNR, we adopt the linear-cavity fiber laser configuration. The capability of this fiber-laser-based sensor system is implemented by tuning a TF located within the laser cavity for interrogating the Bragg wavelengths of all sensing FBGs. The Bragg wavelength shifts induced by the quasi-static strain or temperature drift on the sensing FBGs can be measured by discriminating the lasing wavelength shifts of the system.

\section{EXPERIMENTAL RESULTS AND DISCUSSION}

Fig. 4 shows the experimental setup for our proposed FBG sensor network. We examined three subnetworks in the self-healing ring architecture. Each subnetwork included ten sensing FBGs $\lambda_{i}(i=1,2, \ldots, 10)$. The Bragg wavelengths of the sensing FBGs from FBG $\lambda_{1}$ to FBG $\lambda_{10}$ sequentially were $1538.45,1540.22,1542.86,1544.3,1546.79,1548.47$, $1550.48,1552.43,1554.5$, and $1556.51 \mathrm{~nm}$. All the peak

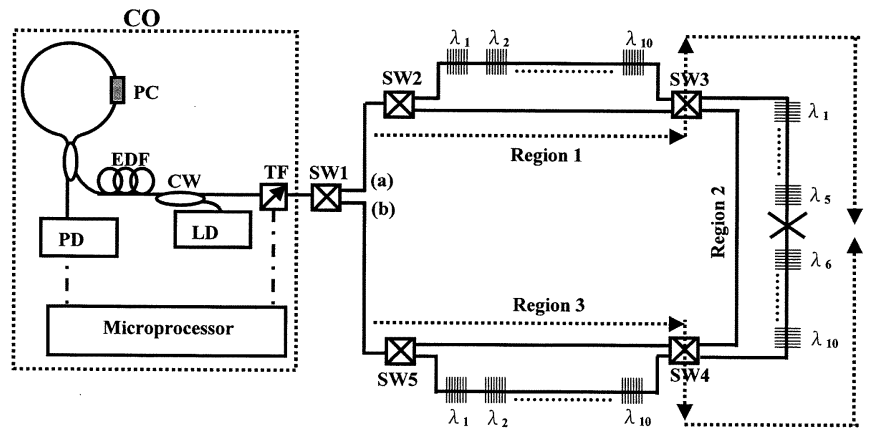

Fig. 4. Experimental setup for the proposed FBG sensor network. We examine three subnetworks in the self-healing ring architecture. Each subnetwork includes ten sensing FBGs $\lambda_{i}(i=1,2, \ldots, 10)$.

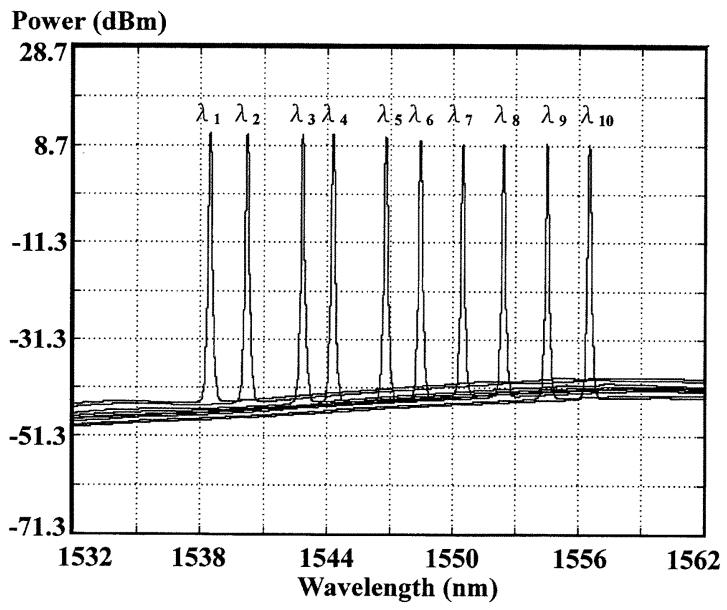

Fig. 5. Output spectra of the linear-cavity fiber laser at different lasing wavelengths.

reflectivities of the FBGs were approximately $99 \%$ and their average 3-dB bandwidth were $0.2 \mathrm{~nm}$. In the central office, the lasing wavelength of the linear-cavity fiber laser was determined by these sensing FBGs in conjunction with the TF. The average 3-dB bandwidth of this TF is $0.37 \mathrm{~nm}$. A 980-nm laser diode with $140 \mathrm{~mW}$ output power pumped the EDF via a $980 / 1550 \mathrm{~nm}$ WDM coupler. In this linear-cavity fiber laser scheme, the coupling ratio of the $2 \times 2$ coupler (C1) for the fiber loop mirror was $30: 70$. The lasing light emerging from this $2 \times 2$ coupler arrived in a photodetector (PD). This signal from the PD finally was fed into a microprocessor for discrimination of the lasing wavelength. With sufficient gain, the system lases once the transmitted wavelength of the filter equals the wavelength reflected from the sensing grating. Thus, the lasing wavelength of the system can be used to accurately measure the strain perturbation imposed on the FBGs. The filter was tuned by using a controller to select the transmitted wavelength over a working range from 1530 to $1560 \mathrm{~nm}$. Hence, the tunable transmitted wavelength of the filter tracked the ten wavelengths of the sensing FBGs $\lambda_{i}$ $(i=1,2, \ldots, 10)$. The output spectra of the linear-cavity fiber laser at different lasing wavelengths are shown in Fig. 5. The average lasing peak power was $9.98 \mathrm{~mW}$ leading to the SNR for the sensing network over $52 \mathrm{~dB}$. When the link failed at Region 2, the FBGs $\lambda_{m}(m=6,7,8,9,10)$ lost their sensing information whenever Sensing Region 2 was selected 


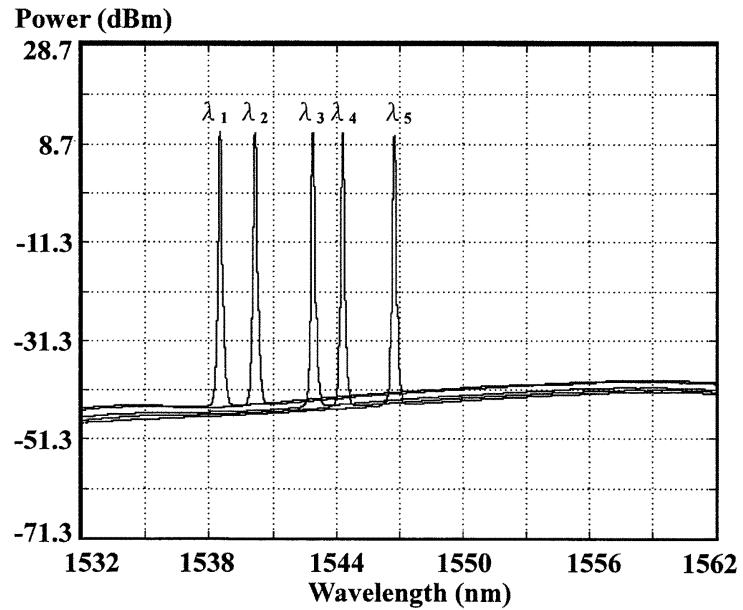

Fig. 6. When the link fails in Region 2, the FBGs $\lambda_{m}(m=6,7,8,9,10)$ loose their sensing information whenever Sensing Region 2 is selected by using a TDM signal.

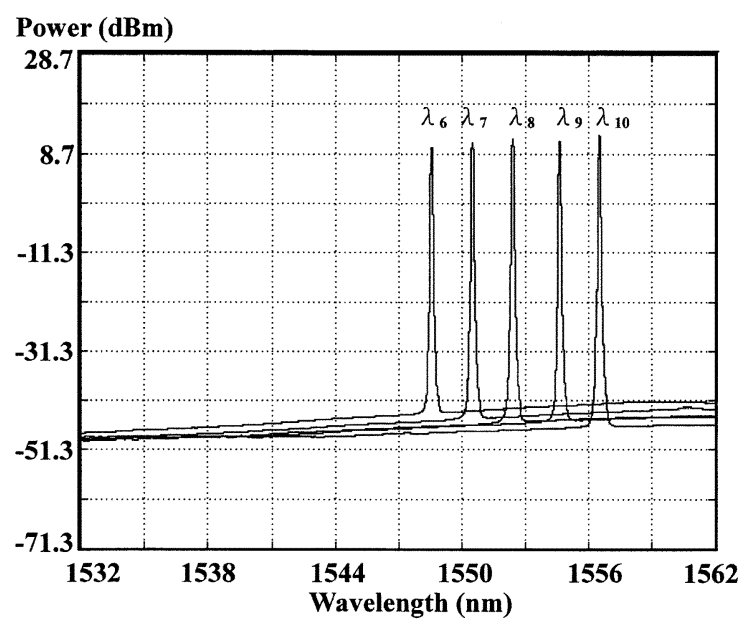

Fig. 7. State (b) of the $1 \times 2$ switch can be modified to reconfigure the fiber link for FBGs $\lambda_{m}(m=6,7,8,9,10)$ that lost the sensing information. The self-healing ring architecture can regenerate the sensing signals from FBGs $\lambda_{m}$ $(m=6,7,8,9,10)$.

by using a TDM signal, as shown in Fig. 6. Nevertheless, state (b) of the $1 \times 2$ switch can be modified to reconfigure the fiber link for FBGs $\lambda_{m}(m=6,7,8,9,10)$ that lost the sensing information, as shown in Fig. 7. Consequently, the self-healing ring architecture can regenerate the sensing signal.

We further concentrate our experiment on the performance of the entire sensing network. Three important issues regarding the practical limitations on our proposed sensor structure are discussed. 1) The lasing power limits the scale of the sensing network. Although the switches and tunable filter facilitate the multiplexing of FBG sensors, they also increase cavity losses and degrade the lasing power. In our experiment, the insertion loss of each $1 \times 2$ switch and $2 \times 2$ switch were below $1 \mathrm{~dB}$; likewise the insertion loss of the filter is $3.42 \mathrm{~dB}$ in a $1530-1560-\mathrm{nm}$ wavelength region. In addition, the reflectivity of the fiber loop mirror is $84 \%$. To estimate the network scale, we assume that the total splicing loss and insertion loss in each subnetwork (including ten FBGs) is $2.3 \mathrm{~dB}$. With a $25-\mathrm{dB}$ gain of our EDF amplifier, the proposed self-healing structure could support 17 subnetworks. 2) Even though the linear-cavity fiber laser with high SNR could support large-scale sensing network, too many subnetworks will lead to poor response time of the system. The operating time on each FBG in the ring architecture are limited by both the TDM signal and the sweeping time of the filter. From 1530 to $1560 \mathrm{~nm}$, the maximum tuning speed of our filter was $920 \mathrm{~ms}$, and the switching time of each switch was $1 \mathrm{~ms}$. Therefore, under the device restrictions of our laboratory, the variation of the quasi-static strain imposed on each FBG cannot be over $0.18 \mathrm{~Hz}$ for this sensor system with three subnetworks. The system response time can be improved by reducing the scale of sensing network, by reducing the number of FBGs in each subnetwork for smaller sweeping time of tunable filter, and basically by using a faster tunable filter. 3 ) The stability of the lasing wavelength and the crosstalk induced by the adjacent FBGs also limit the number of FBG sensors. To avoid such degradation, a feedback-controlled circuitry [4] can be used in the system. For example, we chose $1.44 \mathrm{~nm}$ as the minimum wavelength spacing between the sensing FBGs in our experiment. This wavelength spacing indicates that the maximum strain imposed on each FBG has to be smaller than $598 \mu \varepsilon$ [1] and this quantity should be accurately controlled by the feedback circuitry. According to the above three issues, the optimal self-healing sensor network can be designed by considering the maximum measured strain, the acceptable operating time, and the scale of the ring architecture.

\section{CONCLUSION}

This letter presents a novel self-healing sensor network based on a linear-cavity fiber laser scheme. The sensing network is arranged on the basis of ring architecture. In this letter, we have demonstrated a ten-point FBG sensor based on our proposed configuration and examine its network survivability. Because of the intense lasing power from the linear-cavity fiber laser, the SNR for the sensor network can be over $52 \mathrm{~dB}$. Furthermore, the self-healing function considered in our system can reconfigure the fiber link when a breakpoint suddenly occurs in the sensing network. The experimental results show that the proposed system can facilitate a highly reliable sensing network for a large-scale and multipoint smart structure. We also discuss three practical limitations of this proposed sensor system.

\section{REFERENCES}

[1] A. D. Kersey, M. A. Davis, H. J. Partrick, M. Leblance, K. P. Koo, C. G. Askins, M. A. Putnam, and E. J. Friebele, "Fiber grating sensors," $J$. Lightwave Technol., vol. 15, pp. 1442-1463, Aug. 1997.

[2] L. Talaverano, S. Abad, S. Jarabo, and M. Lopez-Amo, "Multiwavelength fiber laser sources with Bragg-grating sensor multiplexing capability," J. Lightwave Technol., vol. 19, pp. 553-558, Apr. 2001.

[3] L. Zhang, Y. Liu, J. A. R. Wiliams, and I. Bennion, "Enhanced FBG strain sensing multiplexing capacity using combination of intensity and wavelength dual-coding technique," IEEE Photon. Technol. Lett., vol. 11, pp. 1638-1641, Dec. 1999.

[4] Y. Yu, L. Lui, H. Tam, and W. Chung, "Fiber-laser-based wavelengthdivision multiplexed fiber Bragg grating sensor system," IEEE Photon. Technol. Lett., vol. 13, pp. 702-704, July 2001.

[5] J. M. Senior, S. E. Moss, and S. D. Cusworth, "Multiplexing techniques for noninterferometric optical point-sensor networks: A review," Fiber Integr. Opt., vol. 17, pp. 3-20, 1998.

[6] R. Ramaswami and K. N. Sivarajan, Optical Networks: A Practical Perspective. San Francisco, CA: Morgan Kaufmann, 1998. 